\title{
Rheumatic and congenital heart diseases among school children of Khon Kaen, Thailand: declining prevalence of rheumatic heart disease
}

\author{
Arnkisa Chaikitpinyo ${ }^{\text {a }}$, Manat Panamonta ${ }^{\mathrm{a}}$, Yuttapong Wongswadiwat ${ }^{\mathrm{a}}$, Wiboon Weraarchakul ${ }^{\mathrm{a}}$, Ouyporn \\ Panamonta $^{\mathrm{a}}$, Aunejit Panthongviriyakul ${ }^{\mathrm{b}}$, Kaewjai Thepsuthammarat ${ }^{\mathrm{c}}$ \\ ${ }^{a}$ Department of Pediatrics, Faculty of Medicine, Khon Kaen University, ${ }^{b}$ Echocardiographic Laboratory, \\ Faculty of Medicine, Khon Kaen University, 'Clinical Epidemiology Unit, Faculty of Medicine, \\ Khon Kaen University, Khon Kaen 40002, Thailand
}

\begin{abstract}
Background: Rheumatic fever (RF), rheumatic heart disease (RHD), and congenital heart disease (CHD) are still major problems among Thai school children.

Objective: To examine trends in the prevalence of RF/RHD and CHD along with the socioeconomic status of school children in urban Khon Kaen, northeastern Thailand.

Methods: We conducted cross-sectional survey of 8,555 school children aged 5-15 years from 4 schools in urban Khon Kaen from January to March 2006. Pediatric cardiologists examined the school children and all cardiac diagnoses were confirmed by echocardiography. Socioeconomic data were also collected. Schools were divided into high and low socioeconomic status (SES) schools, based on the prevailing levels of parental education and household income. All positive cases of heart disease were followed and reviewed at a university hospital up to December 2013.

Results: Of 8,555 children examined, 2 had RF/RHD, and 10 had CHD. The prevalence of RF/RHD was 0.23 per 1,000 (95\% CI 0.03-0.84), and the prevalence of CHD was 1.2 per 1,000 (95\% CI 0.56-2.15). Prevalence of RF/RHD among urban school children in the center of northeastern Thailand had declined from 1.13 to 0.23 per 1,000 since 1986. The indices of socioeconomic development revealed marked improvement during this 20 year interim. The prevalence of RF/RHD was higher among low SES schools (4.6 per 1,000) compared with high SES schools (0 per 1,000).

Conclusion: There is a low prevalence of RHD in school children in this region compared with the period before 1986.
\end{abstract}

Keywords: Congenital heart disease, heart disease, prevalence, rheumatic fever, rheumatic heart disease

Many previous studies have confirmed that rheumatic fever (RF), rheumatic heart disease (RHD), and congenital heart disease (CHD) are major problems among school children [1-7].

Although rheumatic fever (RF) and rheumatic heart disease (RHD) have virtually disappeared from most parts of the developed world, they continues to be a public health problem in developing countries [7-10]. The prevalence of RF/RHD varies from country to country, within different geographical regions in the same country, and among rural to urban settings. The prevalence also differs within the same region among populations having different ethnic

Correspondence to: Manat Panamonta, MD, Department of Pediatrics, Faculty of Medicine, Khon Kaen University, Khon Kaen 40002, Thailand. E-mail: manat@kku.ac.th origins [10]. Most reported studies of RF/RHD in Thailand originated from large hospital-based tertiary centers [7-9]. The few reported prevalence rates of RF/RHD in school children varied from 1.1-2.1 per 1000 aged 5-15 years in the 1970s and 1980s [3-6]. Epidemiologic data of RF/RHD in Thailand are scarce [3-6] and are not up to date [3-6].

Heart diseases in children, including RHD and $\mathrm{CHD}$, are not generally reported to the public health departments. Therefore, estimating the prevalence in different geographic and climatic areas, in specific age groups, in varying socioeconomic levels, and in different ethnic populations needs to be done by survey. Reliable data of RHD and CHD prevalence are needed for planning and budgeting to provide long-term care for affected children. 
Therefore, a study to determine the epidemiological trend of RF/RHD and its association with changes in socioeconomic status (SES) in school children aged 5-15 years in Khon Kaen was conducted, using the same screening method as was done in the most recent survey conducted in the area in 1986.

\section{Materials and methods Setting}

Thailand is divided into 4 regions; central, northern, northeastern and southern. Khon Kaen province is located in the center of northeastern Thailand at 16.4467 N latitude, 102.833 E longitude, and 167 meters above sea level. According to the 2006 census, the population of Khon Kaen province was approximately 1.7 million. Khon Kaen city is located at the center of Khon Kaen province. Khon Kaen University's Srinagarind Hospital is a large 1,100 bed referral facility located within Khon Kaen city [11]. This study targeted urban school children to study impact of changes in SES on the prevalence of RF/ RHD and CHD and compare findings with a previous study in 1986 (Table 1) [4]. The economic parameters in this geographic region are substantially lower than in Bangkok or in central, northern, or southern regions of Thailand (data from the most recent census).

Thailand has had universal health care coverage since 2001, and people can access health services in their district and, if necessary, be referred for specialist treatment.

\section{Diagnostic criteria}

Mitral regurgitation was characterized by a pansystolic murmur maximal at the apex with radiation to the left axilla. Aortic regurgitation was clinically identified by the presence of an early diastolic murmur heard along the left sternal border [7, 8]. Systolic murmurs were graded on the scale of one to six as described by Levine [12]. Rheumatic fever and rheumatic fever recurrence were diagnosed according to the Jones criteria $[13,14]$.

Table 1. Comparison of socioeconomic characteristics*, health care parameters*, and survey outcomes of the urban area of Khon Kaen city in 1986 and 2006.

\begin{tabular}{lcc}
\hline Characteristics & $\mathbf{1 9 8 6}$ & $\mathbf{2 0 0 6}$ \\
\hline Sample size (urban school children) & 7106 & 8555 \\
Age (years) mean \pm SD, (range) & $9.7 \pm 2.6(7-12)$ & $10.0 \pm 2.8(5-15)$ \\
Sex & & 4352 \\
$\quad$ Male & 4026 & 4203 \\
$\quad$ Female & 3080 & 2226 \\
Economic parameters & & 70.7 \\
$\quad$ Per capita income per year (U.S. dollar)*† & 928 & 96 \\
$\quad$ Employment rate (\%)* & 65.5 & 74.1 \\
Education status & & 7.9 \\
$\quad$ Literacy rate (\%)* & 86 & 99.5 \\
Health indicators & & 18.9 \\
$\quad$ Life expectancy (years)* & 66.4 & 31.5 \\
$\quad$ Crude death rate (per 1000 population)* & 8.1 & 4.2 \\
$\quad$ Health insurance coverage (\%)* & 30.1 & 2 \\
Smoking* (\%) & 25.9 & 10 \\
Alcohol consumption* (\%) & 29.5 & 9 \\
Average number persons per household* & 5.8 & 1 \\
Rheumatic fever, rheumatic heart disease cases & 8 & 2 \\
Congenital heart disease cases & 20 & 19.5 \\
Mitral valve prolapse cases & 8 & \\
History of Kawasaki disease & 0 & \\
History of cardiac arrhythmia & 0 & \\
Percentage of innocent heart murmurs & 36.9 & \\
& & \\
\hline
\end{tabular}

* Data obtained from Khon Kaen municipal office. ${ }^{\dagger}$ Exchange rate of 1 US dollar equal to 31 Thai Baht. 
An acute attack of rheumatic carditis diagnosis was based on auscultatory findings of either mitral regurgitation or aortic regurgitation or both in a patient who had no previous history of mitral or aortic regurgitation. Mild rheumatic carditis was defined as the presence of acute rheumatic carditis without any evidence of cardiomegaly. Moderate rheumatic carditis was categorized as radiological evidence of cardiomegaly in the presence of acute rheumatic carditis without congestive heart failure [9].

Echocardiographic diagnosis of mitral regurgitation was based on the following criteria: presence of a mosaic color regurgitation jet in at least two planes, regurgitating flow sample covering almost the entire systole by pulsed or continuous wave Doppler imaging, and Doppler velocity regurgitation signals with pulsed or continuous waves close to that expected for the Bernoulli-predicted pressure gradient between the left ventricle and left atrium or with a mosaic color jet $>1 \mathrm{~cm}[7,8]$.

RHD was diagnosed clinically in children who had mitral regurgitation, aortic regurgitation, or both [7-8, 15-16]. CHD was defined as a defect in the structure of the heart or great vessels that was present at birth [2, 4].

Mitral valve prolapse was diagnosed when the left ventricle contracted, mitral valve leaflets bulge (prolapse) upward or back into the left atrium. This might cause a murmur [4].

Innocent heart murmurs were defined as grade 1 to grade 3 systolic ejection murmurs, which tended to decrease in intensity in deep inspiration and included vibratory murmurs, pulmonary flow murmurs, systemic flow murmurs, or a soft continuous murmur of venous hum. A child with an innocent murmur had no signs or symptoms of heart disease and had a normal heart.

\section{Study procedures}

Four out of 26 schools located in the urban area of Khon Kaen city were randomly selected for the study. Schools were divided into two high and two low SES schools based on the level of parental education and household income obtained from survey data. The high SES schools had a greater average level of parental education and household income compared with the low SES schools. The crosssectional survey study covered 4 schools located in Khon Kaen city and comprising 8,555 students that were examined between January and March 2006. This study was approved by the Khon Kaen University human research ethics committee. A team of pediatric cardiologists visited the schools to conduct the survey after informing the principal and school nurse, and obtaining the written informed consent from the students and their parents.

This study included all the children in these schools aged 5-15 years. The medical histories relating to cardiovascular symptoms, rheumatic fever, rheumatic heart disease, and Kawasaki disease manifestations were carefully screened and additional information, where needed, was obtained from the students, nurses, or parents. Moreover, the level of parental education and household income were obtained for each student by asking the parents. Among the 8,555 students, 422 (4.9\%) children were absent during examination days. They were revisited on following days and examined. Clinical examinations were performed by pediatric cardiologists with experience in the diagnosis of rheumatic fever, rheumatic heart disease, congenital heart disease, and other heart ailments in children.

The examination included a search for cyanosis and clubbing of fingers, palpation of femoral pulses, and auscultation of the precordial area [4]. Each child was examined in a quiet room in an upright position. A child suspected of having any positive findings was examined in both upright and supine positions. Murmurs were recorded in terms of type, location, transmission, timing, and intensity. Two qualified pediatric cardiologists independently examined the children and the auscultatory diagnoses were based on agreement by both examiners. Children who were considered to have positive findings were referred to Khon Kaen University Hospital for further evaluation. This included chest X-ray imaging, electrocardiography, and echocardiography, and the child was again examined by two pediatric cardiologists. Echocardiographic studies were performed using a Hewlett-Packard Sonos 1000 unit (Hewlett-Packard, Andover, MA, USA). Echocardiographic findings were interpreted independently by two pediatric cardiologists. A complete blood count, erythrocyte sedimentation rate, C-reactive protein test, throat swab culture for group A streptococci, anti-streptolysin $\mathrm{O}$ titer, and antideoxyribonuclease $\mathrm{B}$ titer were performed in each patient who was suspected of having RF. Children designated as having RF or RHD were treated according to a standard international protocol [7-9] and were given secondary antibiotic prophylaxis to prevent streptoccocal infections and recurrent rheumatic fever [7-8]. 


\section{Follow-up}

All positive cases for heart diseases were followed by the pediatric cardiologists of the University Hospital including clinical and echocardiographic review. This was continued until December 2013.

\section{Statistical analysis}

Prevalence is reported as cases per thousand school children. Statistically significant differences were estimated using a chi-square test or Fisher's exact test, and $P<0.05$ was considered as significant.

\section{Results}

A total of 8,555 school children were examined, of which 4,352 (50.9\%) were boys and 4,203 (49.1\%) were girls.

The prevalence of RF/RHD in urban school children in the center of Northeastern Thailand had declined from 1.13 to 0.23 per 1,000 between 1986 and 2006 [4]. The distribution of age, sex, and urban background among study populations in the two survey studies was similar. Indicators of socioeconomic status revealed substantial improvement over a period of 20 years. This was reflected by increase in per capita income, employment rate, literacy rate, life expectancy, and health coverage, and in a decrease in crude death rate (Table 1).

RHD was found in 2 children belonging to low SES schools with a prevalence of 0.2 per 1,000 (95\% CI 0.03-0.84). The prevalence of RF/RHD was higher in the low SES schools (4.6 per 1,000) as compared with high SES schools (0 per 1,000) (Table 2).

The two children with RHD had a history of RF diagnosed and treated at the Khon Kaen University Hospital (Table 3). One child had rheumatic mitral regurgitation and the other child had combined rheumatic mitral and aortic regurgitation.

CHD was found in 10 children with prevalence of 1.2 per 1,000 (95\% CI $0.56-2.15$ ). Isolated ventricular septal defect (VSD) was the most common congenital lesion $40 \%$, followed by atrial septal defect $20 \%$, pulmonary stenosis $10 \%$, VSD with patent ductus arteriosus (PDA) 10\%, tetralogy of Fallot (TOF) $10 \%$, and double outlet right ventricle (DORV) $10 \%$ (Table 4). Five (50\%) of the 10 children including VSD (2 cases), VSD with PDA (1 case), TOF and DORV had indications for corrective surgery. The surgery was performed at Khon Kaen University Hospital and with good outcomes.

Table 2. Comparison of diseases at high or low socioeconomic status schools

\begin{tabular}{lccc}
\hline Heart diseases (number of students) & High SES s & Low SES s & $\boldsymbol{P}^{*}$ \\
\hline Number of students & 5,187 & 3,368 & \\
Rheumatic fever and Rheumatic heart disease (2) & 0 & 2 & 0.16 \\
Congenital heart disease (10) & 5 & 5 & 0.53 \\
Mitral valve prolapse (9) & 4 & 5 & 0.33 \\
History of Kawasaki disease (1) & 1 & 0 & 0.999 \\
History of cardiac arrhythmia (2) & 1 & 1 & 0.999 \\
Innocent heart murmur $^{\dagger}(\%)$ & 19.2 & 19.9 & 0.42
\end{tabular}

SESs $=$ socioeconomic status schools, ${ }^{*} P<0.05$ was considered as significance, ${ }^{\dagger}$ an innocent heart murmur is not a disease

Table 3. Clinical courses of rheumatic fever and patients with rheumatic heart disease.

\begin{tabular}{|c|c|c|c|c|c|}
\hline $\begin{array}{l}\text { Age/Sex/Date of the } \\
\text { first ARF attack }\end{array}$ & $\begin{array}{l}\text { Presentation of } \\
\text { the first attack }\end{array}$ & $\begin{array}{l}\text { Echocardiogram } \\
\text { of the first attack }\end{array}$ & $\begin{array}{l}\text { Secondary } \\
\text { prophylaxis }\end{array}$ & $\begin{array}{l}\text { Recurrence/ Date/ } \\
\text { Clinical presentation }\end{array}$ & $\begin{array}{l}\text { Follow-up } \\
\text { status/Date }\end{array}$ \\
\hline $\begin{array}{l}5 \text { y/female/ Nov } \\
2001\end{array}$ & $\begin{array}{l}\text { Carditis; MR and } \\
\text { AR }\end{array}$ & MR, AR, and TR & Regular & No & $\begin{array}{l}\text { MR, and AR/ } \\
\text { Nov } 2013\end{array}$ \\
\hline 12 y/male/Jan 2004 & $\begin{array}{l}\text { Carditis and } \\
\text { Polyarthritis; MR }\end{array}$ & MR and AR & Irregular & $\begin{array}{l}\text { Yes/Jan 2005/MR and } \\
\text { AR and polyarthritis }\end{array}$ & $\begin{array}{l}\text { Aortic valve } \\
\text { repair/May } 2008\end{array}$ \\
\hline
\end{tabular}

$\mathrm{ARF}=$ acute rheumatic fever, $\mathrm{MR}=$ mitral regurgitation, $\mathrm{AR}=$ aortic regurgitation, $\mathrm{TR}=$ tricuspid regurgitation 
Table 4. Congenital heart diseases found during the 2006 survey

\begin{tabular}{lc}
\hline Type of congenital heart diseases & Number of patients \\
\hline Ventricular septal defect & 4 \\
Atrial septal defect & 2 \\
Pulmonary stenosis & 1 \\
VSD with patent ductus arteriosus & 1 \\
Tetralogy of Fallot & 1 \\
Double outlet right ventricle & 1 \\
\hline
\end{tabular}

VSD = ventricular septal defect

Other heart diseases were asymptomatic mitral valve prolapse in 9 (1.1 per 1,000), Kawasaki disease (by history) in 1 ( 0.1 per 1,000), and cardiac arrhythmia of supraventricular tachycardia (by history) in 2 (0.2 per 1,000$)$. An innocent heart murmur was found in $19.5 \%$ of the children studied. The prevalence of innocent heart murmurs showed no significant variation by age, gender, or by socioeconomic characteristics.

\section{Discussion}

The present study is the first report that documents the apparent five-fold decline in prevalence of RF/RHD from 1.13 to 0.23 per 1,000 urban school children in the center of Northeastern Thailand, between 1986 and 2006 [4]. The two-point estimation of the prevalence of RF/RHD in the same study area and using similar screening methods, after a gap of 20 years, suggests that a decline of prevalence of RF/ RHD is seen with improvement in living standards and health care services resulting in decreasing acquisition of group A streptococci (Table 1). Moreover, this study also found that the prevalence of RF/RHD was higher in the low SES schools (4.6 per 1,000) compared with high SES schools (0 per 1,000) (Table 2).

Most reported data concerning RF/RHD from Thailand are hospital-based studies from large tertiary centers [7-9]. Major tertiary care centers in Thailand are located in provincial capitals. Only a few vulnerable people with rheumatic fever, such as school-aged children seek primary medical treatment from such referral centers [7-9]. Because of these limitations, a school-based study is the most worthwhile method to ascertain the actual epidemiology of RF/RHD in this most vulnerable population [3-5]. Almost all previous population-based epidemiologic surveys relied on the careful clinical examination of school-aged children, with confirmation of clinically suspected cases by echocardiography. Surveys during 1970s to 1980s showed the prevalence of rheumatic heart disease to be approximately 1.12.1 cases per 1000 school children in Thailand [3-6] and 1.8 to 11 cases per 1,000 school children in other developing countries [15]. Although recent reports show the high sensitivity of echocardiography in screening RHD, and that this more sensitive screening can add more cases of RHD compared with clinical examination alone, controversy concerning the specificity of echocardiographic definitions remains [16].

The prevalence of CHD among school children in this 2006 study of 1.2 per 1,000 (95\% CI $0.56-$ 2.15) was comparable to reports from other parts of Thailand, which varied from $0.4-4.6$ per 1,000 school children [2-6]. VSD was the most common CHD (Table 4). The majority of these congenital lesions required active management or follow-up. These findings highlight the importance of input from pediatric cardiologists to facilitate the correct diagnoses of CHD.

This study had a number of limitations. First, the study was confined to one center in the northeastern region and results cannot be generalized to other parts of Thailand. Second, the actual time of occurrence of $\mathrm{RF} / \mathrm{RHD}$ is uncertain. Temporal changes in disease prevalence would have been best described by continuous data collection. Third, because we did not study the profile of group A streptococci strains prevalent in the study area, the change in rheumatogenic virulence of the streptococcal strains in the study area over a period of time (20 years) could be associated with the decline in the RF/RHD cases.

\section{Conclusion}

The prevalence of RF/RHD has declined by almost five-fold over the last 20 years. This appears to be largely related to improvement in socioeconomic 
status and the healthcare delivery system. The role of changes in the rheumatogenic characteristics of the prevalent streptococcal strains in the study area cannot be ruled out. Policy intervention to improve living standards and healthcare facilities and awareness can go a long way in reducing the morbidity and mortality burden of RF/RHD. Prospective longitudinal observational studies to collect more data are warranted.

\section{Acknowledgement}

The authors would like to thank Dr. James A. Will for his editorial assistance. This study was supported by a grant from Khon Kaen University. All authors have no conflict of interest to report.

\section{References}

1. Yu CH, Lue HC, Wu SJ, Chen MR. Heart disease screening of school children in Taiwan. Arch Pediatr Adolesc Med. 2009; 163:233-7

2. Cholkraisuwata E, Buddharaksaa Y, Sayasathidb J. Congenital heart disease in elementary school children in rural Thailand: the role of the trained non-cardiologist. Asian Biomed. 2013; 7:287-91.

3. Pongpanich B, Dhanavaravibul S, Limsuwan A. Prevalence of heart disease in school children in Thailand: a preliminary survey at Bang Pa-in. Southeast Asian J Trop Med Pub Hlth. 1976; 7:91-4.

4. Panamonta M, Chaikitpinyo A, Klungboonkrong V, Tantisirin C, Bhuripanyo K. Heart diseases in school children: a preliminary survey in the Khon Kaen municipal area. Srinagarind Hosp Med J. 1986; 1: 253-7.

5. Vichitbandha P, Podhipleux P, Prachuabmoh C, Nana A, Kongsamran S, Rungpitarungsi B, et al. Epidemiology of heart disease in school children in rural and urban areas. J Med Assoc Thai. 1981; 64: 1-10.

6. Sayasathid J, Tantiwongkosri K, Somboonna N. Unrecognized congenital heart disease among Thai Children. J Med Assoc Thai 2009; 92:356-9.
7. Panamonta M, Chaikitpinyo A, Auvichayapat N, Weraarchakul W, Panamonta O, Pantongwiriyakul A. Evolution of valve damage in Sydenham's chorea during recurrence of rheumatic fever. Int J Cardiol. 2007; 119:73-9.

8. Panamonta M, Chaikitpinyo A, Kaplan EL, Pantongwiriyakul A, Tassniyom S, Sutra S. The relationship of carditis to the initial attack of Sydenham's chorea. Int J Cardiol. 2004; 94:241-8.

9. Panamonta M, Settasatian N, Kaplan EL, Chaikitpinyo A. Serum cholesterol levels in patients with acute rheumatic fever. Am J Dis Child. 1993; 147:732-6.

10. Carapetis JR. Rheumatic heart disease in developing countries. N Engl J Med. 2007; 357:439-41.

11. Panamonta M, Chaikitpinyo A, Lumbiganon P, Panamonta O, Auvichayapat N, Wongswadiwat Y, et al. Historical assessment of diphtheritic myocarditis from a hospital in northeastern Thailand. Asian Biomed. 2014; 8:475-84

12. Levine SA. The systolic murmur: its clinical significance. JAMA. 1933; 101:436-8.

13. Special Writing Group of the Committee on Rheumatic Fever, Endocarditis, and Kawasaki Disease of the Council on Cardiovascular Disease in the Young of the American Heart Association. Guidelines for the Diagnosis of Rheumatic Fever, Jones Criteria, 1992 Update. JAMA. 1992; 268:2069-73.

14. American Heart Association. Jones criteria (revised) for guidance in the diagnosis of rheumatic fever. Circulation. 1984; 69:204A-8.

15. Negi PC, Kanwar A, Chauhan R, Asotra S, Thakur JS, Bhardwaj AK. Epidemiological trends of RF/RHD in school children of Shimla in north India. Indian J Med Res. 2013; 137:1121-7.

16. Marijon E, Celermajer DS, Tafflet M, El-Haou S, Jani DM, Ferreira B, et al. Rheumatic heart disease screening by echocardiography: the inadequacy of World Health Organization criteria for optimizing the diagnosis of subclinical disease. Circulation. 2009; 120:663-8. 